\title{
Sobrado da Convenção em Itu na antiga Rua do Carmo (atual Rua Barão do (taim): uma pesquisa documental
}

\section{Anicleide Zequini}

Museu Republicano Convenção de Itu-MP/USP

As datas atribuídas à construção do "Sobrado da Convenção"

O primeiro Guia do Museu Republicano "Convenção de Itu" foi publicado após vinte e três anos em que a instituição havia sido aberta ao público. Affonso de E. Taunay, idealizador do Museu e organizador da primeira exposição procurou, em janeiro de 1946, explicar esse descompasso: "Esperei, porém que os trabalhos de azulejamento do grande saguão do edifício estivessem concluídos para realizar tal desideratum agora" 1 .

Este azulejamento, corresponde a um conjunto de azulejos pintados sobre temas e personalidades relacionadas à História de ltu, que haviam sido encomendados ao pintor Antonio Luiz Gagni para compor a exposição inaugural. Na mesma ocasião foram adquiridos também, de diversas procedências, móveis e objetos que, segundo Taunay, pudessem oferecer aos visitantes a "idéia do que eram o mobiliário e a ornamentação de uma casa rica brasileira pelas vizinhanças de 1870"2.

A escolha de azulejos, e não de outro material, para a realização daquelas pinturas foi determinada a partir da constatação de que este tipo de material estava presente no revestimento externo do edifício desde 1867, conforme inscrição encontrada na platibanda do prédio "E como víssemos azulejado o frontispício do Museu, entendemos que tal decoração poderia vantajosamente ser feita por azulejamento das paredes do vestíbulo" 3 .

É também nesta publicação que pela primeira vez encontramos mencionada a data de 1867 associada à construção do edifício. "Acha-se o Museu Republicano Convenção de ltu instalado no belo e vasto sobradão sito à rua Barão do Itaim (outrora do Carmo) casarão construído em 1867 por José de
1. E.TAUNAY.Affonso de. Guia do Museu Republicano "Convenção de Itu". São Paulo: Indústria Gráfica Siqueira, 1946. p. 3 .

2. Id., p. 11.

3. Id., p. 12. 
4. Id., p. 11.

5. MAIA, Tom. et al. Itu: quatro séculos de história. São Paulo: Expressão Editorial, 1995. p. 130-131.

6. LISANTI FILHO, Luiz Comércio e capitalismo: o Brasil e Europa entre o fim do século XVIII e o início do século XIX. O exemplo de três Vilas: Itu, Porto Feliz e Campinas. 1962. p. 109.Tese (Doutorado) - IFLCH, Universidade de São Paulo, São Paulo, 1962.
V. Almeida Prado e, pouco depois, por ele vendido a seu irmão Carlos V. de Almeida Prado $4 . "$

Esta periodicidade - incorporada posteriormente em textos relacionados à História do Museu e à cidade de ltu - permaneceu presente até a década de 90 do século XX. Um destes trabalhos, intitulado ltu - Quatro Séculos de História, comemorativo ao 120 Aniversário da "Convenção Republicana" de 1873 e publicado em 1995, constitui exemplo dessa constatação. Assim, num dos verbetes encontramos a seguinte informação sobre o Museu Republicano "No ano de 1867, os irmãos Carlos e José de Almeida Prado, construíram na rua Barão do Itaim um elegante sobrado, com sua fachada revestida de azulejos" ${ }^{\prime \prime}$.

Mas examinando o entorno deste edifício veremos que a data de sua construção pode ser anterior ao ano de 1850.

Itu e a economia açucareira

Em 1774 havia na vila de ltu 779 casas, dois conventos de religiosos (Carmo e São Francisco) e a lgreja Matriz ainda em construção. Situada na área compreendida pelo "quadrilátero do açúcar", Itu destacava-se pela presença de inúmeros engenhos de açúcar, responsáveis por 1/3 do açúcar fabricado na Capitania de São Paulo.

A riqueza gerada principalmente por este produto estimulou o crescimento populacional da vila, no período compreendido entre o final do século XVIII e início do XIX. Além desta questão demográfica, a economia açucareira intensificou o comércio, as artes e a urbanização. ${ }^{\circ}$ Data deste período a inauguração da igreja Matriz (1780), a igreja do Carmo (1782) e melhoramentos urbanos, como a pavimentação das ruas em 1790.

Podemos observar, numa das primeiras plantas urbanas da vila, executada por Sá e Faria em 1774, a presença de um traçado urbano formado por quadras retangulares delimitadas por ruas e becos. Característica que se apresenta relativamente preservada até o presente (FIGURA 1).

Nesse documento aparecem destacadas algumas ruas da localidade, como a Rua do Carmo, atualmente denominada Barão do ltaim, e áreas ocupadas por edificações, com destaque para a localização da "igreja Nova" latual Matriz da Candelária) e da igreja-convento do Carmo.

\section{A Rua do Carmo (atual Rua Barão do ltaim)}

As primeiras casas de sobrado de ltu, conforme pudemos constatar através da pesquisa documental, foram construídas entre a virada do século XVIII e o início do século XIX. As mais antigas localizavam-se nas áreas correspondentes ao alinhamento da Rua Direita (atualmente Rua Paula Souza), Largo da Matriz (atual Praça Padre Miguel) e Rua do Carmo (atual Rua do Barão do Itaim), locais escolhidos para residências dos senhores-de-engenho.

A concentração de pessoas enriquecidas na Vila sobretudo pelo açúcar 


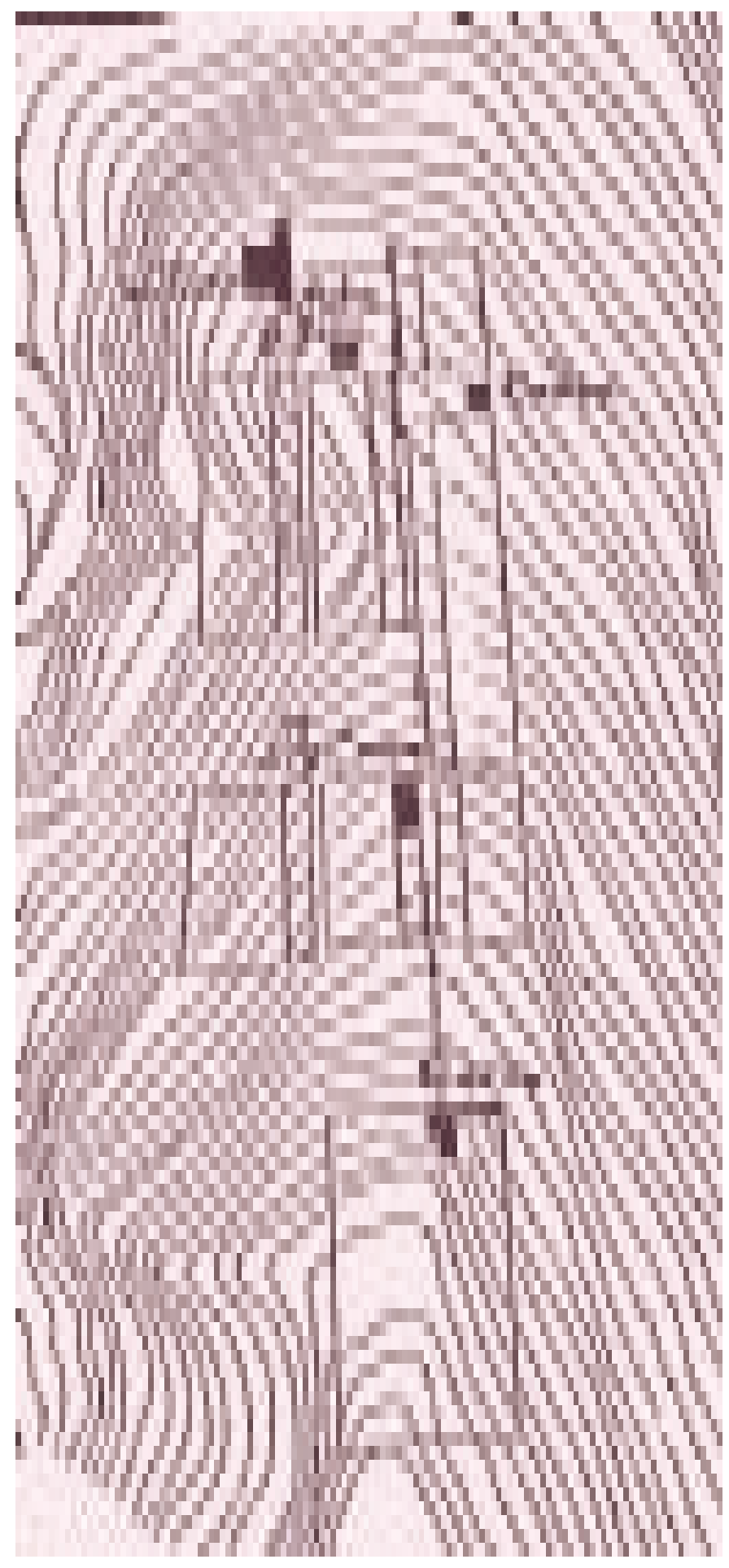

FIGURA 1 - Planta Urbana da Vila de ltu executada pelo Engenheiro J.C. de Sá e Faria. 1774 In: BASTOS, Maria Antonieta de Toledo Ribeiro. A Cidade de ltu: "Berço da República", um estudo de Geografia Urbana até a I República (1930). Doutorado, SP:IFLCH-USP, 1997. 
7. DEAN, Warren. A Fábrica São Luiz de Itu: um estudo de arqueologia industrial. Anais de História, Assis, ano VIII, p. 11, 1976.

8.O tenente Elias, irmão de Josepha Maria de Góes Pacheco, era proprietário do Engenho Grande (atualmente Chácara do Rosário), herdado de seu pai, o sargento-mor Antonio Pacheco da Silva, sertanista em Cuiabá. Como outros senhores-de-engenho que habitavam Itu nessa mesma época, Elias encaminhou seus filhos para estudar Direito. Um deles, Fernando Pacheco Jordão, estudou em Portugal na Universidade de Coimbra, enquanto Francisco de Assis Pacheco Jordão e José Elias Pacheco Jordão estudaram em São Paulo.

9. Relação e Avaliação de Bens. In: Inventário de D. Antonia Fausta Rodrigues Jordão, ACCI-MR, $1^{\circ}$. Of., 1825, fl. 8. Sobre a família Pacheco consultar: ASSIS PACHECO BORBA, Frederico de. Pacheco de Itu: antepassados e descendentes de Elias Antonio Pacheco da Silva. São Paulo: Vaner Bícego, 1977.247 p.

10. Inventário do Dr. Fernando Pacheco Jordão. ACCI-MR, $1^{\circ}$. Of. 1859 fl. 9

11. Livro de Notas, 1836 fls. 77 v.e de 1838 , fls. 145 .

12. Id., 1838 fls. 145.

13. Id., ibid.

14. Elias Antonio Pacheco Chaves, formado em Direito,residia em São Paulo. Foi fazendeiro de café e exerceu alguns cargos políticos no Estado de São Paulo, como o de deputado-geral. Casado com Anézia Prado, faleceu em 1903.
1829, à Câmara de ltu, um pedido para que a localidade fosse dividida em duas áreas administrativas: a "Vila Velha" e a "Vila Nova" (FIGURA 2).

A Vila Nova constituída pelo Pátio da Matriz (atual Praça Padre Miguel), Rua do Carmo (atual Rua Barão do Itaim) e adjacências refletiu, como poderemos observar, o período de declínio econômico do açúcar, que se estendeu até 1840. Nesta época o capital e parte da população de ltu começaram a se deslocar para as zonas cafeeiras do "Oeste Paulista"7. Como parte desta constatação observamos que ao longo do século XIX somente foram construídos três sobrados na Rua do Carmo, dois deles anteriores a 1830.

\section{sobrado de Josepha Maria de Góes Pacheco}

Encontramos em 1825 a primeira referência sobre a edificação de um sobrado na Rua do Carmo (atual Rua Barão de Itaim). Pertencia este imóvel a Josepha Maria de Góes Pacheco, proprietária, em ltu, de um grande número de escravos e de engenho de açúcar.

Nesse mesmo ano, observamos que na área correspondente ao Pátio da Matriz (atual Praça Padre Miguel) estavam sendo construídos, sob a iniciativa do tenente Elias Antonio Pacheco da Silva (senhor-de-engenho), dois outros sobrados (um deles na esquina do Pátio da Matriz) e uma casa térrea (vizinha à de Josepha Maria de Góes Pacheco) $)^{8}$ (FIGURAS 3, 3a e 4).

pilão e:

primeiro desses sobrados tinha as paredes construídas de taipa de

[...] 7 janelas na frente do largo da Matriz e 4 janelas na frente da rua do Carmo, com beiral forrado, ditas janelas assentadas, menos as folhas que só se acham feitas, como também as competentes grades de ferro e o assoalho limpo por pregar, sem repartimento algum e só com algum taboado de forro limpo e alguns batentes de dentro aparelhados, cunhais de pedra por assentar, escada do quintal quase concluída. ${ }^{9}$

Em 1859 este sobrado passou a pertencer a Fernando Pacheco Jordão, e no final do século XIX, à família Pereira Mendes. ${ }^{10}$

O outro sobrado anexo a essa construção, edificado na Rua do Carmo (atual Rua Barão do Itaim), encontrava-se no mesmo estado do anterior, faltando apenas a construção das escadas dos fundos, área onde se encontrava a cozinha. Vendido em 1836 ainda inacabado, "cobertas de telhas, com o vigamento, portas e janelas de frente, e as de cima com grades de ferro" para a Loja Maçônica Ituana, foi revendido em 1838 para Fernando Pacheco Jordão ${ }^{11}$. Dessa forma, os sobrados (da esquina do Pátio da Matriz e da Rua do Carmo) passaram a pertencer novamente à família Pacheco Jordão'2.

Quanto à casa térrea unida a esses sobrados, também em construção em 1828, contava nessa época apenas com portas e janelas assentadas, não possuía repartição interna e era coberta de telhas ${ }^{13}$. Na partilha realizada naquele ano, coube este imóvel a D. Antonia Fausta Rodrigues Jordão, e com o falecimento dela, a casa passou para seu filho Elias Antonio Pacheco Chaves, que pelo menos até 1890 apareceu como seu proprietário ${ }^{14}$.

Nota-se que esse conjunto de edificações somente iria desaparecer do cenário urbano de ltu na década de 70 do século XX, ocasião em que foram demolidas aquelas edificações para a construção de um outro edifício (FIGURA 5). 


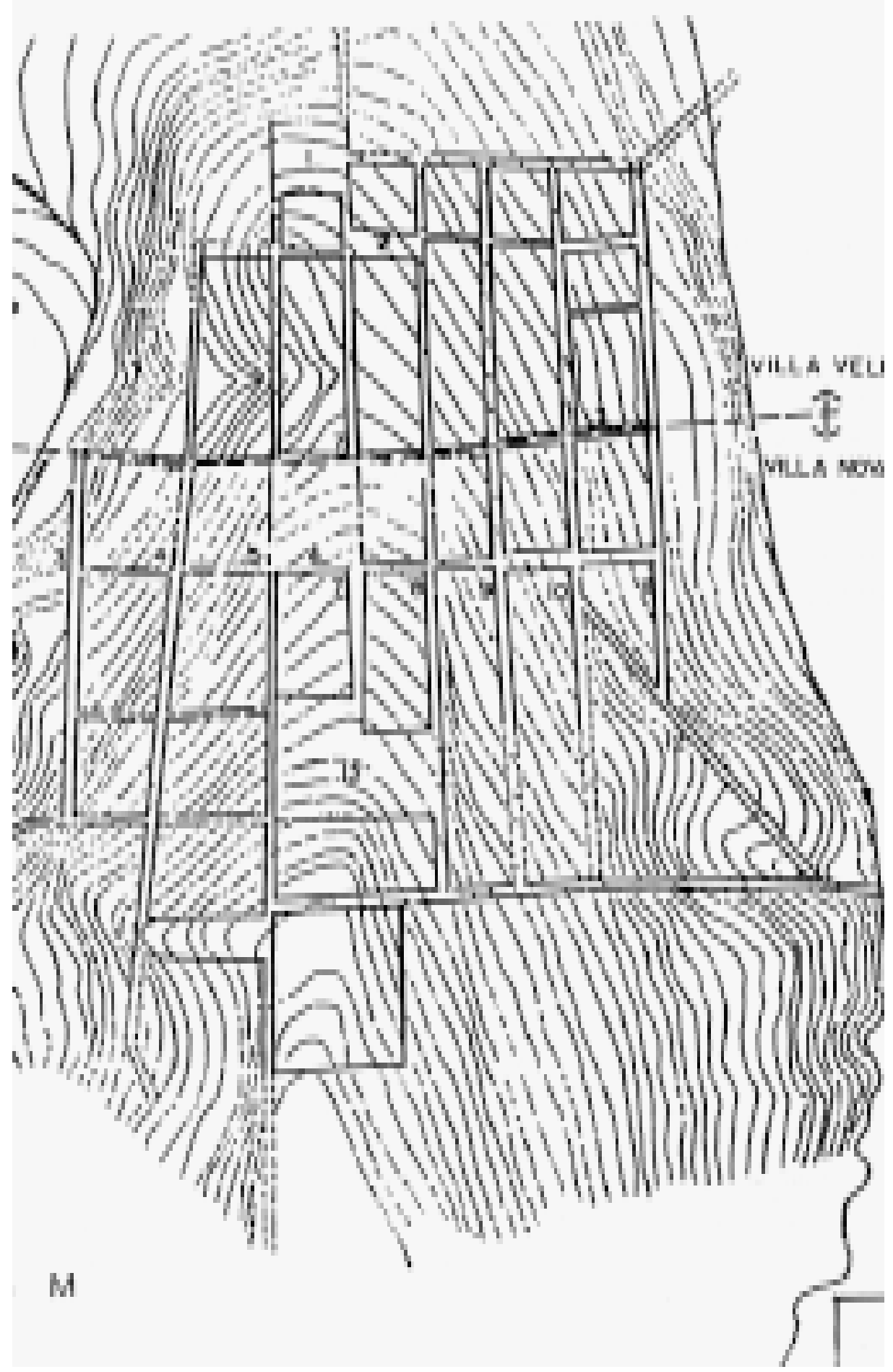

FIGURA 2 - Planta Urbana de Itu, com as delimitações da "Vila Velha" e "Vila Nova", 1830. In: TOSCANO, João Walter. Itu/Centro Histórico: estudo para preservação, Mestrado, S'P. FAU-USP, 1981. 


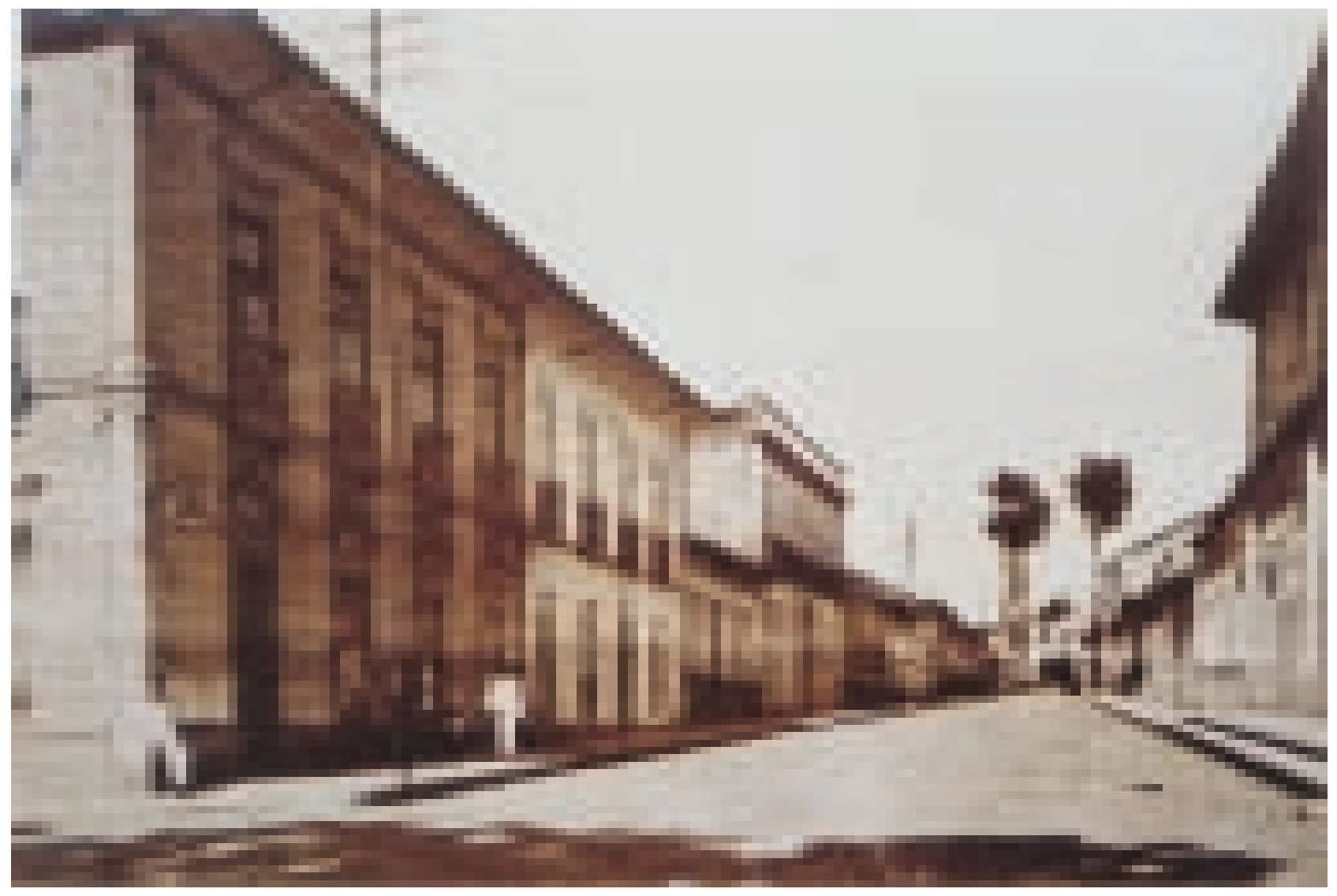

FIGURA 3 - Rua do Carmo (atual Rua Barão do Itaim). 1940

Em primeiro plano, o conjunto de edifícios (2 sobrados) e uma casa térrea, construídos na primeira metade do século XIX pelo Tenente Elias Antonio Pacheco. Acervo do Museu

Republicano Convenção de ltu - MP/USP. 


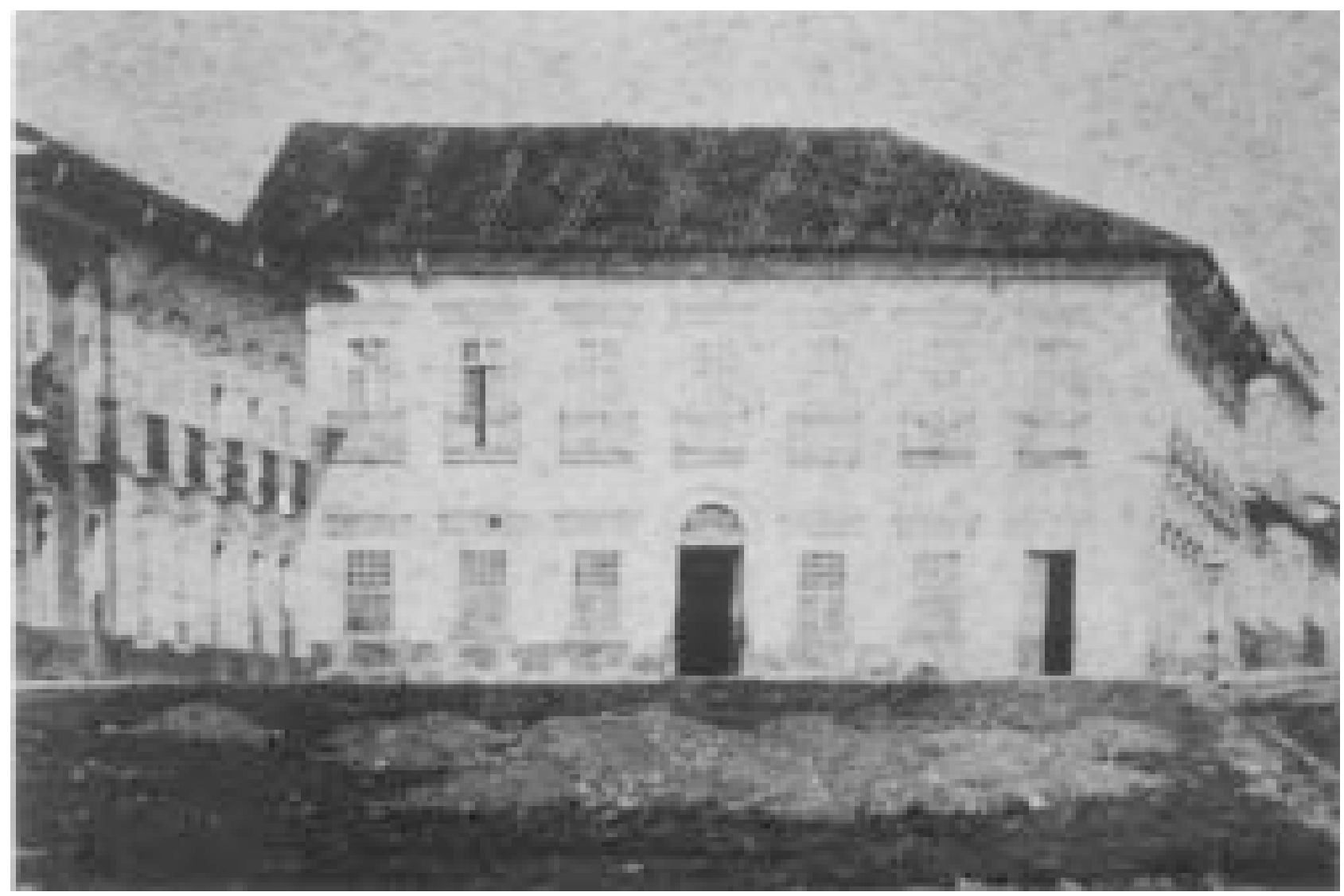

FIGURA 3a - Largo da Matriz (atual Praça Padre Miguel). 1899

Em destaque a fachada do sobrado construído em meados do século, pelo Tenente Elias Antonio Pacheco. Acervo do Museu Republicano Convenção de ltu - MP/USP. 


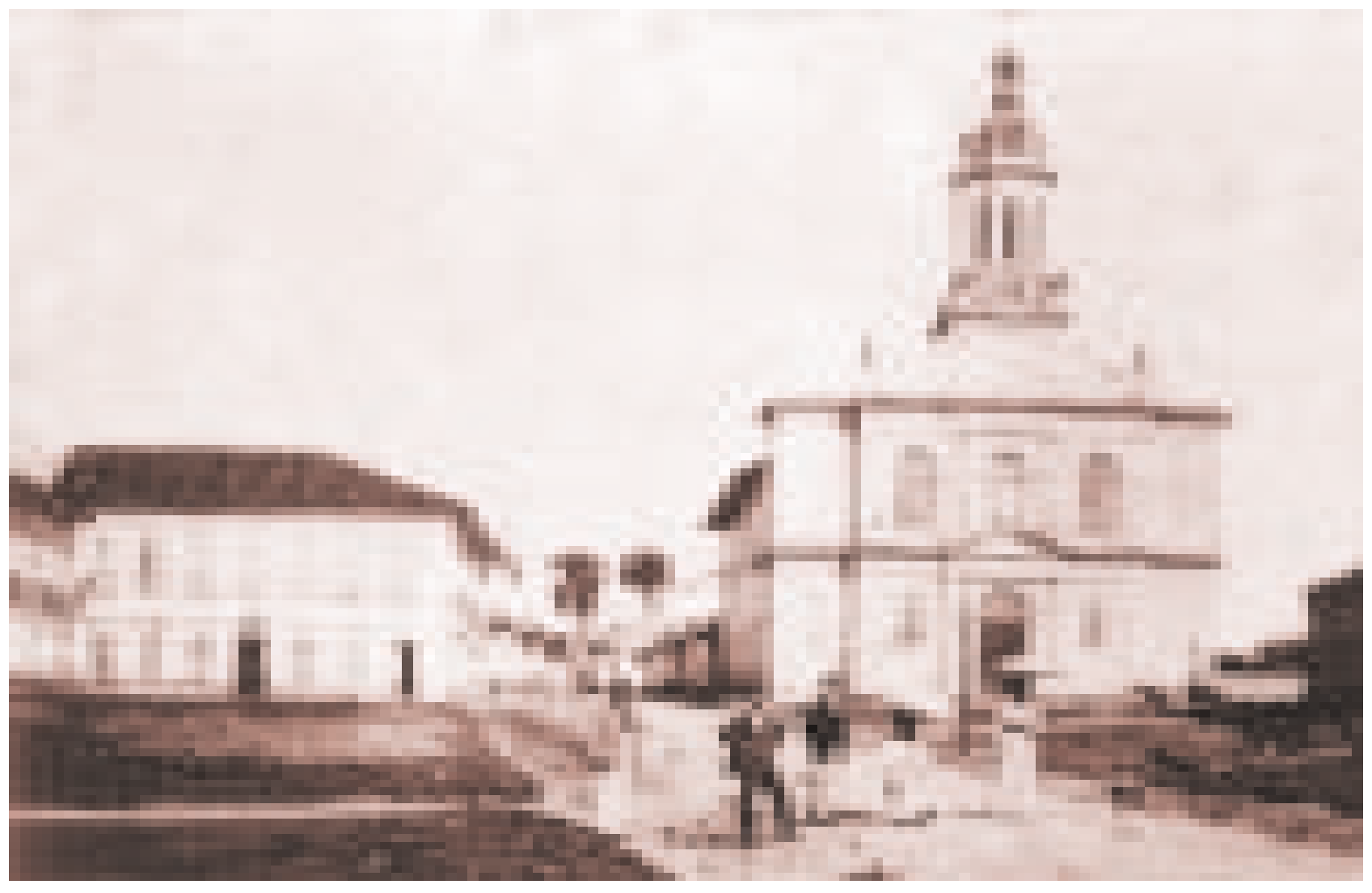

FIGURA 4 - Largo da Matriz (atual Praça Padre Miguel). 1899. Acervo do Museu Republicano 


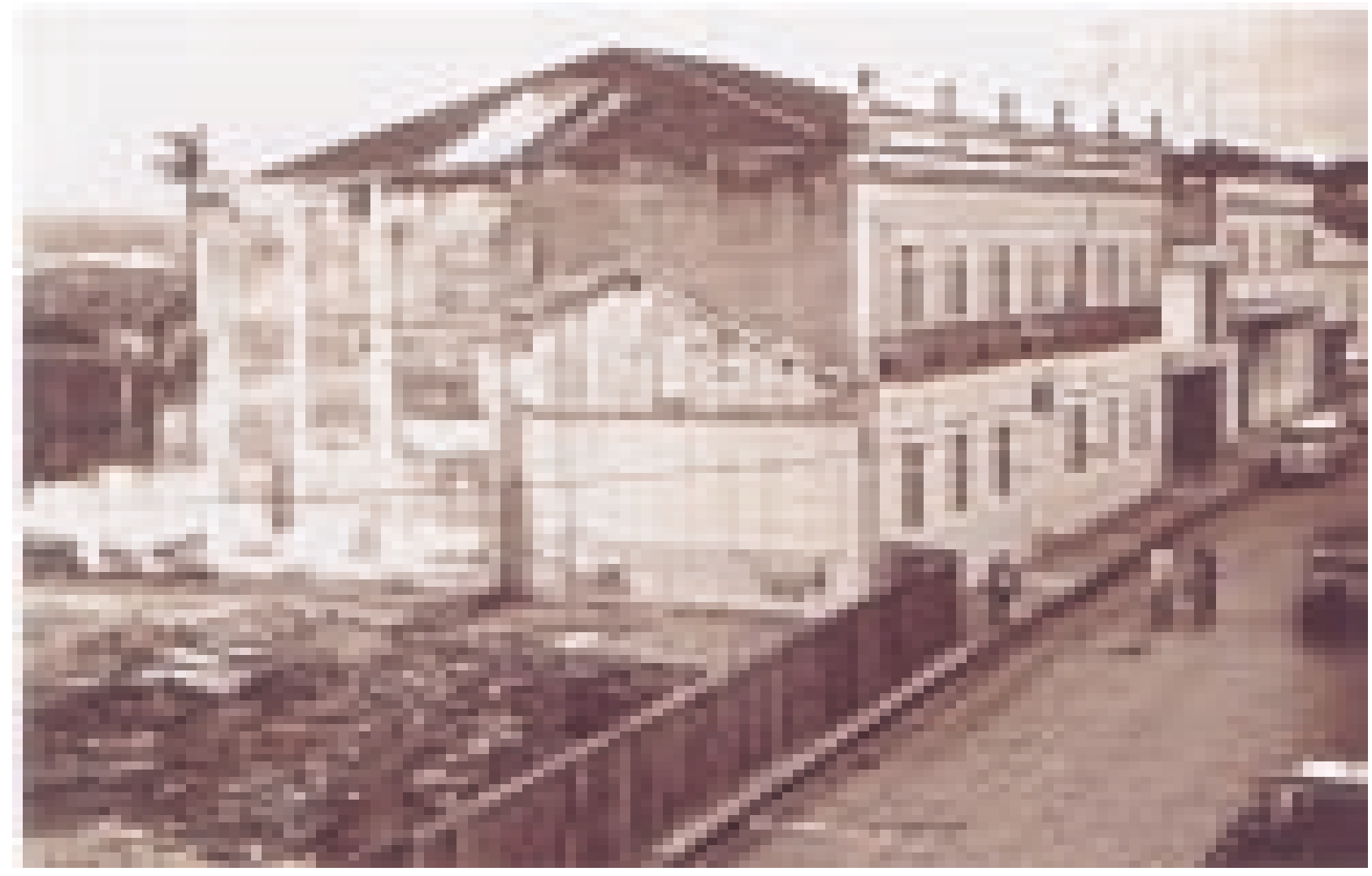

FIGURA 5 - Rua do Carmo (atual Barão do Itaim). Década de 70 (séc. XX)

Em primeiro plano, a demolição dos antigos sobrados construídos no início do século XIX. Acervo do Museu Republicano Convenção de lłu - MP/USP. 
15. Inventário de Josepha Maria de Góes Pacheco. ACCI-MR, $1^{\circ}$. Of., cx. 36A, 1829. fl. 31 .

16. Id.

17. Para análise sobre esta questão ver: BARBUY,Heloisa Maria Silveira. $A$ C $i$ dade-Exposição: comércio e cosmopolitismo em São Paulo, 1860-1914 (estudo de história urbana e cultura matéria). 2001. p. 39-49.Tese (Doutorado) Faculdade de Arquitetura e Urbanismo, Universidade de São Paulo, São Paulo, 2001.

18. Inventário de Josepha Maria de Góes Pacheco. ACCI-MR, $1^{\circ}$. Of., cx. 36 A, 1829.fl. 31

19. Francisco de Almeida Prado, filho de Lourenço deAlmeida Prado,adquiriu sesmarias em Itu em meados do século XVIII. O sítio Esperança, em 1835, possuía engenho de açúcar com moendas movidas por força animal (bois).
Josepha Maria de Góes Pacheco e o primeiro sobrado

Em 1825 D. Josepha, moradora da Rua do Carmo, foi também neste local a primeira proprietária de um sobrado. Em 1829 este imóvel, conforme pode ser constatado em seu inventário, estava passando por algumas reformas como a colocação de "molduras para o forro"15. Uma descrição mais completa deste sobrado, de 1828, indica que ele possuía "7 janelas em cima e 7 portas embaixo,"16 fato que faz supor que a parte inferior tenha sido destinada ao comércio, conforme "a tradição ibérica e a conjugação de comércio e residência ${ }^{17 "}$.

A divisão do sobrado de Josepha e a constituição de duas moradias

Em 1832 o inventário de Josepha Maria de Góes Pacheco não havia sido finalizado. Contudo, os herdeiros já haviam entrado em acordo sobre a partilha de alguns de seus bens, entre eles, o sobrado da Rua do Carmo. Dois de seus filhos estavam requerendo a propriedade: Francisca Xavier da Fonseca e seu irmão, Joaquim Manoel Pacheco da Fonseca.

Para solucionar esta questão, Joaquim pediu um parecer, transcrito abaixo, a dois peritos sobre a viabilidade de ser feita uma divisão da propriedade. Formuladas as perguntas eles as responderam.

Pergunta: "Se tendo de separar a parede divisória por cima da escada, que arranjo deve ter?" Resposta:

Que a escada deve ficar no corredor de baixo da quarta janela e seguirá o primeiro andar, e o segundo lugar oposto do segundo andar da escada. Observarão a importância da parede divisória da casa até o quintal, entrando barrotes, ripas, moirão, caibros de goaratan e jornais de escravos, pela quantia de 41 \#360 Rs.

Pergunta: "E quanto à importância da parede divisória da casa e quintal?"

Resposta: "Declarou que a parede de cima não perde parede alguma, e que a de baixo não perde alcova porque deve mudar a escada para o lugar competente. Declarou mais que a mudança da escada avalião feitio e pregos 18 \#000 Rs."

Pergunta: "Se por causa da mesma divisa a morada de cima vem perder uma parede ou não, e se a de baixo perde uma alcova."

Resposta: "Declarou que se fazer a escada nova para a casa do lado de cima - 57\#200 Rs ${ }^{18} . "$

Com parecer favorável, o sobrado foi dividido, provavelmente em 1832, formando duas habitações com extensões desiguais, sendo que a unidade mais próxima ao Pátio da Matriz resultou composta de 4 janelas (em cima) e 4 portas (embaixo), portanto a outra unidade ficou com as restantes 3 janelas (em cima) e 3 portas (embaixo).

A primeira unidade ficou para os herdeiros órfãos, enquanto a outra unidade ficou para Francisca Xavier da Fonseca. 
20. Livro de Notas, 1839. fl. 64

21. Francisco de Almeida Prado foi casado duas vezes.A primeira,com Maria Dias Pacheco; ver: Inventário,ACCI-MR, $1^{\circ}$. Of., cx. 43, 1835. e depois com Anna Joaquina Vasconcellos Noronha; ver:Inventário de Anna Joaquina Vasconcellos Noronha. ACCI-MR, $2^{\circ}$. Of., cx. 51, 1866. Em 1855, Francisco de Almeida Prado possuía em Itu duas fazendas com engenho de açúcar (São Borja e Capoava) e outras duas glebas de terra (Sítios Curimã e Braiyá). Havia adquirido na localidade de Limeira, na região do "Oeste Paulista",mais duas outras propriedades rurais: o Sítio Paraguassu, com engenho de açúcar e plantação de café, e uma gleba de terra denominada Sítio Palmeira.A busca por terras novas marcou uma transição de interesse econômico que identificou alguns grandes proprietários rurais de Itu quando, a partir de 1850 , o café - que apresentava maior lucratividade em relação ao açúcar - passou a ser difundido na região da "depressão periférica" paulista.

22. Inventário de Anna Joaquina de Vasconcellos Noronha.ACCI-MR, $2^{\circ}$. Of., cx. 51, 1866.

23. A Acta da Convenção de Itu. In: E. TAUNAY, Affonso. Op. cit. p. 61-63.

24. O imóvel entregue como parte do pagamento era um sobrado existente na "Travessa da Matriz, canto com a Rua da Palma". Livro de Notas, $2^{\circ}$ Of., 1890.fls. 15-17. pelo assentamento de uma porta principal (FIGURAS 6 e 7).

Tendo sido este sobrado novamente partilhado em 1894, coube a propriedade aos sobrinhos de Francisco de Paula Leite de Barros, que passaram a ser seus novos proprietários. Em 1922, a Fazenda do Estado de São Paulo adquiriu o edifício para a instalação do Museu Republicano. Após a realização 


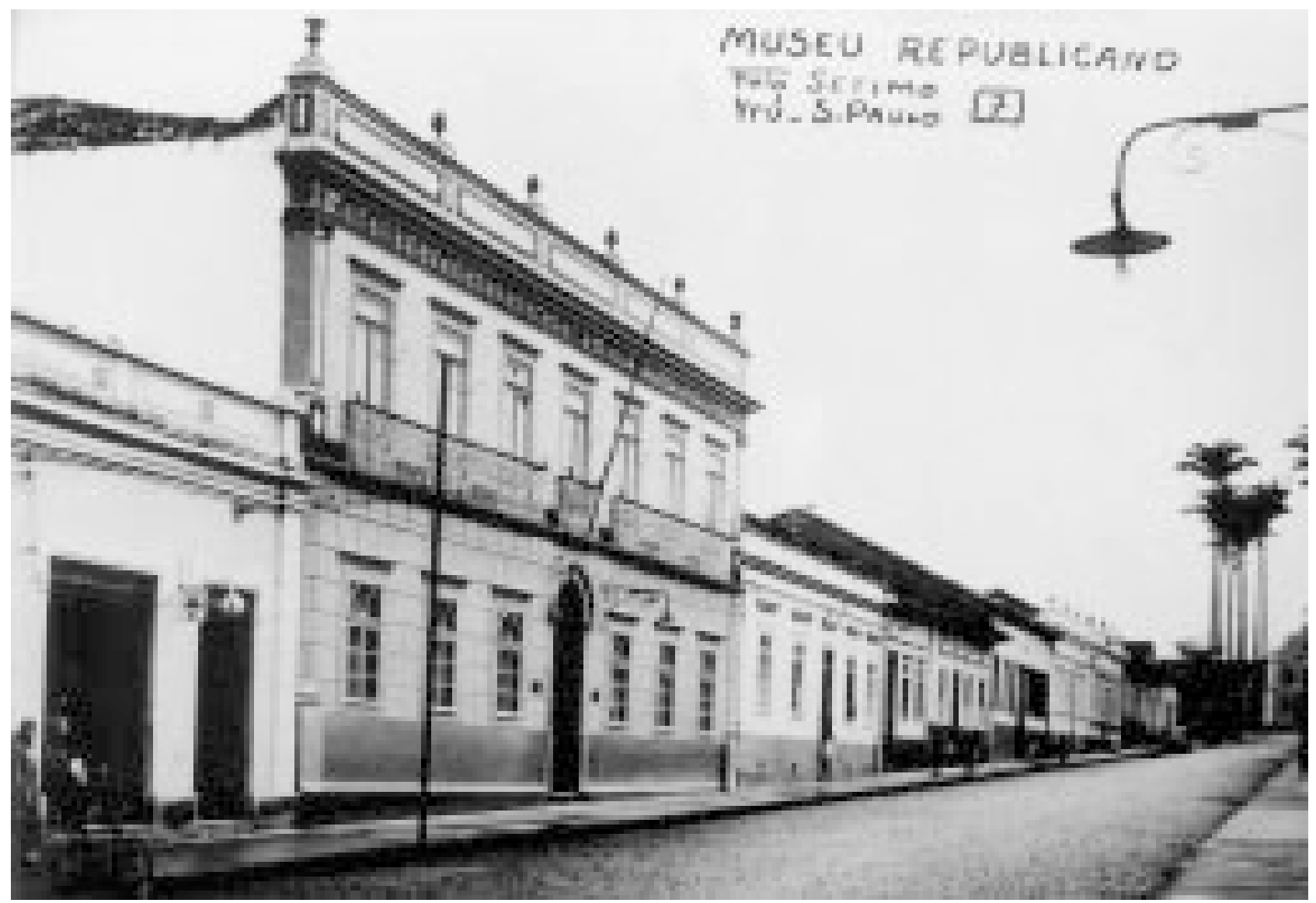

FIGURA 6 - Prédio do Museu Republicano "Convenção de ltu" ( Itu-SP). Década de 20 (séc. XX.) Parte inferior do edifício ( 6 janelas e 1 porta) em substituição às 7 portas existentes no século XIX. E a parte superior (7 portas), em substituição às 7 janelas do século XIX. Acervo do Museu Republicano Convenção de ltu - MP/USP. 


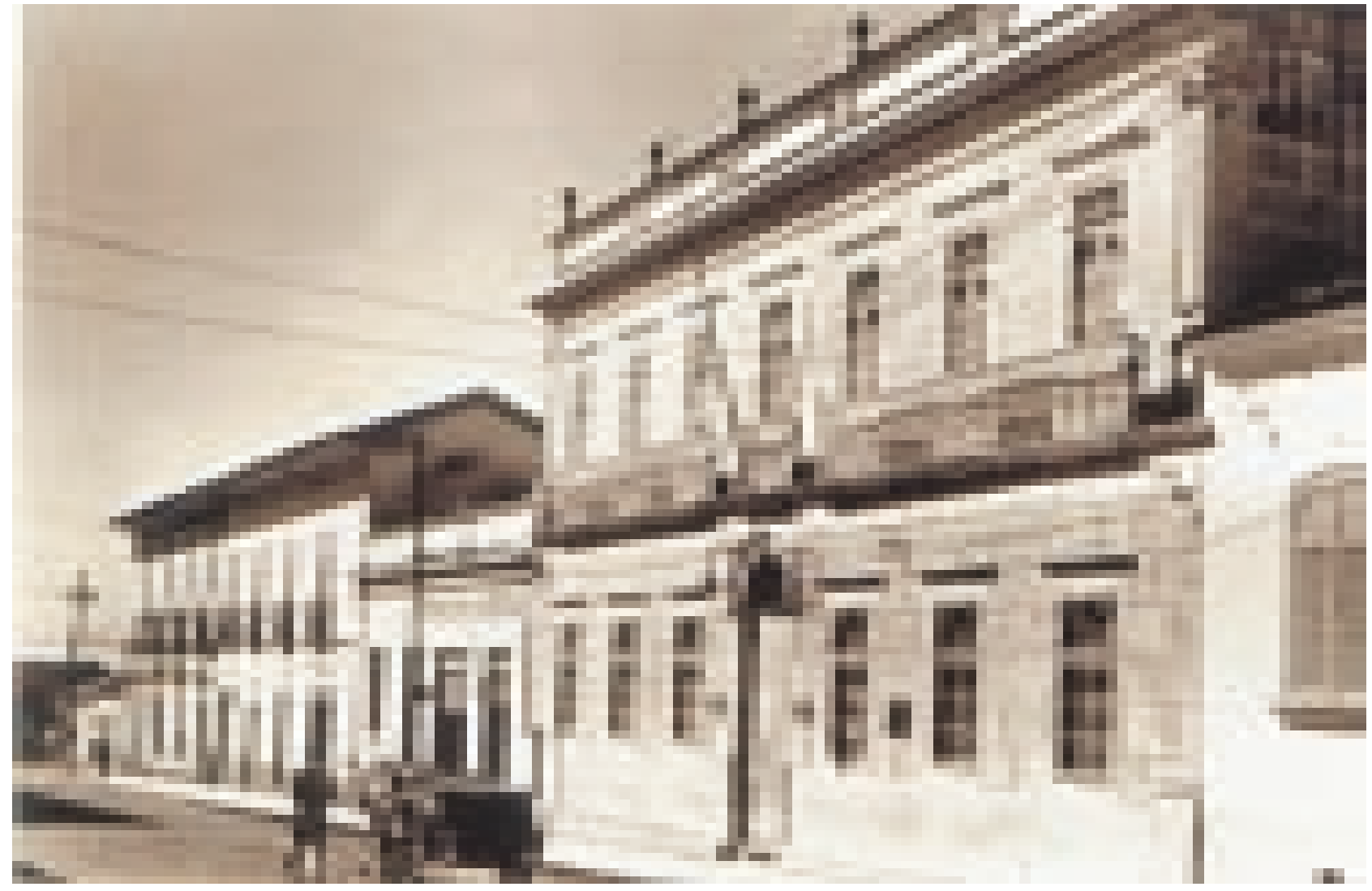

FIGURA 7 - Prédio do Museu Repubilcano Convenção de ltu (ltu-SP). Década de 20 (séc. XX). Outro ângulo da Rua do Carmo (atual Rua Barão do Itaim). Acervo do Museu Republicano Convenção de ltu - MP/USP. 
de uma ampla reforma sob a direção de Affonso de E. Taunay, inaugurou-se na cidade de ltu, em 1923, o Museu Republicano "Convenção de ltu", como anexo do Museu Paulista da Universidade de São Paulo.

Assim, a partir de todas as informações pesquisadas, podemos supor que a data de construção do edifício que abriga o Museu seja bem anterior àquela que the vem sido atribuída, e que o ano de 1850 marca a presença de uma das famílias moradoras daquele edifício: os Almeida Prados, que receberam os Convencionais de 1873.

\section{FONTES:}

Arquivo do Museu Republicano "Convenção de Itu"/MP/USP

Inventários. $1^{\circ}$ Of., cx. 30, 36 A, 43 A, 69, 74 A

Inventários. $2^{\circ}$ Of., cx. 51

Arquivo e Museu Histórico Municipal de Itu

Livro de Notas.Anos: 1836,1838,1839

Cartório de Notas de Itu

Livro de Notas.Ano: 1890

\section{REFERÊNCIAS}

ASSIS PACHECO BORBA, Frederico de. Pacheco de Itu: antepassados e descendentes de Elias Antonio Pacheco da Silva. São Paulo:Vaner Bícego, 1977. 247 p.

BASTOS, Maria Antonieta de Toledo Ribeiro. A Cidade de Itu: berço da república, um estudo de geografia urbana até a $1^{\text {¿ }}$ república (1930). Tese (Doutorado) - Faculdade de Filosofia Letras e Ciências Humanas, Universidade de São Paulo, 1997.356p.

BARBUY, Heloisa Maria Silveira. A Cidade-Exposição: comércio e cosmopolitismo em São Paulo, 1860-1914 (estudo de história urbana e cultura matéria). Tese (Doutorado) - Faculdade de Arquitetura e Urbanismo, Universidade de São Paulo, São Paulo, 2001. 298p.

DEAN, Warren.A Fábrica São Luiz de Itu: um estudo de arqueologia industrial. Anais de História, Assis, ano VIII, 1976.

LISANTI FILHO, Luiz. Comércio e capitalismo: o Brasil e Europa entre o fim do século XVIII e o início do século XIX. O exemplo de três Vilas: Itu, Porto Feliz e Campinas. Tese (Doutorado) IFLCH, Universidade de São Paulo, São Paulo, 1962. 157p. 
MAIA,Tom. et al. Itu: quatro séculos de história. São Paulo: Expressão Editorial, 1995. 149p.

TAUNAY, Affonso de E. Guia do Museu Republicano “Convenção de Itu”. São Paulo: Indústria Gráfica Siqueira, 1946.73p.

TOSCANO, João Walter. Itu/Centro Histórico: estudos para preservação. Dissertação (Mestrado), Faculdade de Arquitetura e Urbanismo, Universidade de São Paulo, 1981. 175p.

Artigo apresentado em 11/2003. 
It deals with the presentation of fourteen Miguelzinho Dutra's water-colours unknown to the general public and the researchers. These images come to complement the image repertoire produced by the painter which are at USP's Museu Paulista.

KEYWORDS: Collection. Landscape. Painting.

Anais do Museu Paulista. São Paulo. N. Sér. v. 10/11.p.149-166 (2002-2003)

Ornamentação do Museu Paulista para o Primeiro Centenário: construção de identidade nacional na década de 1920

Miyoko Makino

Nas últimas décadas, vários estudos enfocaram o Museu Paulista, as coleções, o edifício-monumento e a gestão Afonso de Escragnole Taunay, contribuindo para o desenvolvimento do conhecimento histórico e do patrimônio arquitetônico, artístico e cultural de São Paulo. A ornamentação do Museu, organizada por Taunay, para os festejos do Primeiro Centenário da Independência, em 1922, composta essencialmente de pinturas e esculturas, visando narrar o período colonial até a Independência, é o foco deste estudo. As fontes permitem entender o caminho da seleção dos temas, a execução e a disposição das obras, em vários níveis, no Saguão, Escadaria e Salão de Honra. Os anos iniciais da gestão Taunay (1917 - 1945) foram propícios para a execução da ornamentação, visto o apoio oficial e os recursos financeiros obtidos, inclusive da sociedade paulista. A conclusão da ornamentação, no entanto, demorou duas décadas, pela exigüidade de verbas, após as festividades do Centenário. A ornamentação foi dada por concluída por Taunay, com a colocação da última ânfora na Escadaria, em 1931, e a publicação do Guia da Secção Histórica do Museu Paulista, em 1937. No entanto, as últimas pinturas foram feitas e incorporadas nos anos 60 .

PALAVRAS-CHAVE: Museu Paulista. História do Brasil. Iconografia.

Anais do Museu Paulista. São Paulo. N. Sér. v. 10/11.p.167-195 (2002-2003).

Ornamentation of Museu Paulista for the Fist Centennial: construction of national identity in the 1920's

Miyoko Makino

In the last decades, several studies have focused Museu Paulista, the collections, the monument building and Afonso de Escragnole Taunay's term, helping for the development of the historical knowledge and the architectural, artistic and cultural patrimony of São Paulo. The museum's ornamentation, organized by Taunay, for the commemorations of the First Centennial of Independence, in 1922, composed essentially of paintings and sculptures, aiming to narrate the colonial period up to the independence, is the focus of studies. The sources allowed us to understand the way the themes were selected, the making and the disposition of the works, in various levels, in the Hall, the Stairway and the Honour Room. The initial years of Taunay's term (1917 - 1945) were propitious for the making of the ornamentation, given the official support and the financial resources obtained, including the ones from the society of São Paulo. The finalization of the ornamentation, nevertheless, took two decades, due to the exiguity of the income, after the Centennial festivities. The ornamentation was granted as concluded by Taunay, with the placing of the last amphora on the Stairway, in 1931, and the publishing of the Guide of the Historical Section of Museu Paulista (Guia da Secção Histórica do Museu Paulista), in 1937. However, the last paintings were made and incorporated in the 1960's. KEYWORDS: Museu Paulista. History of Brasil. Iconography.

Anais do Museu Paulista. São Paulo. N. Sér.v. 10/11.p.167-195 (2002-2003).

O Sobrado da Convenção em Itu na antiga Rua do Carmo (atual Rua Barão do Itaim): uma pesquisa documental

Anicleide Zequini Rossi

O texto refere-se aos resultados de uma pesquisa documental (inventários post-morten e Livro de Notas) referente a trajetória do edifício em que está instalado o Museu Republicano na Cidade de Itu-SP, extensão do Museu Paulista-USP. Assim, a partir de todas as informações pesquisadas podemos supor que a data de construção do edifício, que abriga o Museu, seja bem anterior a 1850, ano que the tem sido apontado em diversos texto. Este ano registra sim, a presença de uma das famílias moradoras daquele edifício: os Almeida Prado que receberam os Convencionais de 1873.

PALAVRAS-CHAVE: Museu Republicano Convenção de Itu- MP/USP. Itu, História. Itu, Museu. Almeida Prado, Família. 
The two-storey house where Itu Convention was held, in the old Rua do Carmo, Inowadays Rua Barão do ltaim): a documental work of research

Anicleide Zequini Rossi

The text is about the results of a documental work of research (post-mortem inventory and Book of Notes) regarding the trajectory of the building in which The City of Itu (SP)'s Republican Museum (Museu Republicano na Cidade de ltu-SP) is installed, an extension of Museu Paulista - USP. Therefore, from all the pieces of information researched we can infer that the construction day of the building that shelters the museum, is a lot earlier than 1850, year which has been considered as such in many essays. This year registers, in fact, the presence of one of the families that lived in that building: the Almeida Prados that hosted the Conventionals of 1873.

KEYWORDS: Museu Republicano Convenção de Itu - MP/USP. Itu, History. Itu, Museum. Almeida Prado, Family.

Anais do Museu Paulista. São Paulo. N. Sér. v. 10/11.p.197-211 (2002-2003).

De casa a museu: 80 anos do Museu Republicano Convenção de ltu

Jonas Soares de Souza

O texto apresenta um panorama geral do processo de criação do Museu Republicano "Convenção de ltu" na década de 1920. Idealizado pelos republicanos paulistas, o projeto da instituição explorou o valor simbólico da "Convenção de ltu" e o significado do lugar de sua realização como recurso para ampliar a legitimidade histórica da hegemonia do Partido.

PALAVRAS-CHAVE:Arquitetura. Museu. Memória. Comemoração.

Anais do Museu Paulista. São Paulo. N. Sér. v. 10/11.p.213-225 (2002-2003)

From home to museum: 80 years of the Republican Museum (Museu Republicano) ltu Convention

Jonas Soares de Souza

This article presents a general overview of the creation process of Museu Republicano "Convenção de ltu" in the decade of 1920. Idealized by republicans from São Paulo, the institution's project has exploited the Convenção de ltu's symbolic values and the meaning of place where it was made as a resource to widen the historical legitimacy of the Party hegemony.

KEYWORDS:Architecture. Museum. Memory. Celebration.

Anais do Museu Paulista. São Paulo. N. Sér. v. 10/11.p.213-225 (2002-2003)

O Serviço de Objetos do Museu Paulista

Adilson José de Almeida, Angela Maria Gianeze Ribeiro, Heloisa Barbuy, Margarida Davina Andreatta Aborda a organização do Serviço de Objetos do Museu Paulista - curadoria de acervos e sistema de informação - e suas linhas de pesquisa: 1. Formação das coleções. 2. Formas alternativas de dinheiro. 3. Cultura visual no século XIX: museus exposições industriais e cidades / Comércio, industrialização e cultura material. 4. Arqueologia histórica e industrial. 5. Vida militar e cultura material.

PALAVRAS-CHAVE: Curadoria e pesquisa. Numismática. Coleção de veículos. Coleções militares. Cultura visual. Industrialização. Anais do Museu Paulista. São Paulo. N. Sér. v. 10/11.p.227-257 (2002-2003)

The Department of Object Collections of Museu Paulista

Adilson José de Almeida, Angela Maria Gianeze Ribeiro, Heloisa Barbuy, Margarida Davina Andreatta It focuses the organization of the Department of Object Collections of Museu Paulista - curatorship and information system - and its research directions: 1. Formation of the collections. 2. Money alternative forms. 3. Visual Culture in the 19th century: museums, industrial exhibitions and cities / Commerce, industrialization and Material Culture. 4. Historical and Industrial Archaeology. 5. Military life and Material Culture.

KEYWORDS: Curatorship and Research. Numismatic. Collection of Vehicles. Collection of Military Objects. Visual Culture. 